\title{
Cashmere production, harvesting, marketing and processing by nomads of Iran - A review
}

\author{
Hamid Reza Ansari-Renani
}

\begin{abstract}
Iran together with Afghanistan is the third largest producer and exporter of cashmere in the world, after China and Mongolia. Nomads have a big share in producing Iranian cashmere. This study reviews the status of cashmere production, harvesting, marketing and processing by nomads of Iran in order to evaluate sustainability and to identify some constraints limiting productivity. The quality of Iranian cashmere, being long and highly curved, ranks third after China and Mongolia. The principal centres for the gathering and rough sorting of raw cashmere produced by nomads are Baft, Sirjan, Mashad and Birjand, with Mashad continuing as the centre of the Iranian cashmere industry. Nearly all the cashmere produced is bought directly from the nomads by middlemen and is dehaired and exported to Europe and China without major value addition.
\end{abstract}

Keywords: Nomad; Goats; Cashmere quality; Combing; Marketing

\section{Introduction}

Of the 25 million goats in Iran, 5 million are cashmereproducing and the remaining goats are meat and milk types (Ansari-Renani et al. 2013a; Ansari-Renani 2004). Nomads (ashayer) keep $40 \%$ of all goats in habitats covering about $59 \%$ of the country. Nomads keep goats mainly for selling live animals to local markets and for home consumption of meat, but cashmere is also a major source of income for Raeini, Birjandi, Nadushan, Abadeh and Abasabadi goat breed keepers. The major portion of Iranian cashmere is produced by Raeini goats kept by nomads in Kerman Province (Ansari-Renani et al. 2012, 2013a).

The most important determinants of animal fibre quality are the species that produce it and the diameter of the fibre, as previously described for Iranian cashmere (AnsariRenani et al. 2011a, b; Ansari-Renani 2012). The fleece of cashmere goats are double coated and consist of two major fibre types: coarse, medullated guard hair and fine, nonmedullated insulative down fibre (Allain 1992; Mitchell et al. 1991; Nixon et al. 1991; Ryder 1966; Carter and Clark 1943; Petrie 1995). In early spring, as a result of inactivity of secondary follicles, Iranian cashmere goats start a sequential, bilaterally symmetric pattern of shedding their fine undercoat fibres, commencing on the neck, chest and

\footnotetext{
Correspondence: hansarirenani@gmail.com
}

Animal Science Research Institute, Karaj, Iran shoulders and spreading to the back and rump (AnsariRenani 2001).

The quality of Iranian cashmere being long and highly curved (Ansari-Renani et al. 2012, 2013a; McGregor and Postle 2004; McGregor 2007) ranks third in price after China and Mongolia (Schneider 2012). At present, no price differential is paid to nomads for producing fine cashmere, and the producers are not aware of world market prices for different cashmere quality classes (Ansari-Renani et. al. 2013). Almost all cashmere is exported without any added value through processing. As a result of the marketing system, producers do not achieve good prices and have little incentive to produce better quality cashmere. This study reviews the present status of Iranian nomad cashmere goat production, harvesting, marketing and processing and the potential for improving cashmere industry.

\section{Methods}

In-depth open interviews and group discussions were used for acquiring qualitative data. Interviews and discussions were conducted from August to December 2011, mainly in the eastern regions with key cashmere industry actors in Iran: producers, buyers and processors of cashmere. Three different structured questionnaires for producers, buyers and processors were completed, to compile information regarding quality and quantity of

\section{国}

(C) 2015 Ansari-Renani. Open Access This article is distributed under the terms of the Creative Commons Attribution 4.0 International License (http://creativecommons.org/licenses/by/4.0/), which permits unrestricted use, distribution, and reproduction in any medium, provided you give appropriate credit to the original author(s) and the source, provide a link to the Creative Commons license, and indicate if changes were made. 
cashmere, selling season/month, factors important to making decisions on cashmere prices, means of transport, kinds of payment, types of agreement with buyer/seller, market accessibility, type of sorting/storage, packaging, processing machines: scouring, garneting, dehairing, combing, challenges, education, training and customs.

Seven short periods of field work were conducted to interview 40 cashmere farmers in each of the Baft and Birjand regions in Kerman and South Khorasan provinces respectively. Three short periods of field work in December 2011 were also conducted to interview 15 middlemen in the main marketing cities of Kerman, Sirjan, Birjand and Mashad. Fieldwork was also conducted to interview the owners of cashmere mills in Semnan and Mashad Provinces.

\section{Information on prices and marketing margins}

Price information along the value chain was obtained from the different sources (actors) along the value chain, and cross-checked with each other. Prices for raw cashmere were obtained from nomads, district, provincial and national level assemblers and the exporters, while margins were calculated accordingly. Export prices for raw and dehaired cashmere were obtained from processors and exporters. Wholesale and final garment prices were obtained from different websites. The volume of marketed cashmere and corresponding number of goats was mainly derived from available literature, the national census and official statistics.

\section{Discussions}

\section{Cashmere goat breeds}

Nomads play an important role in the country's goat production, mainly because they keep $40 \%$ of Iran's goat population. Approximately $70 \%$ of goats in Iran are of mixed breeds and their crosses, which are mainly kept for meat production, while other types are known for their cashmere (Raeini, Birjandi, Abadeh and Nadoushan), mohair (Markhoz), milk (Najdi) and meat (Tali, Adani and Native black) production (Ansari-Renani et al. 2012, 2013a).

The major portion of Iranian cashmere is produced in the eastern part of the country, mainly by two breeds of goat: Raeini and Birjandi (Baluchi) in Kerman and South Khorasan Provinces, respectively. However, Raeini goats, which are mainly kept by nomads, are the most important cashmere-producing breed in terms of population and thus the volume of cashmere produced.

\section{Cashmere harvesting}

\section{Combing goats to avoid cashmere loss}

In parts of Iran, cashmere is harvested during the three- to six-week spring period when the goats are moulting or by collecting the moulted fibres from the ground and bushes
(Ansari-Renani et al. 2012, 2013a; Ansari-Renani 2013). Cashmere is harvested by nomads using combs at the beginning of spring in the south-eastern provinces of South Khorasan, Khorasan Razavi and parts of Sistan and Baluchistan. In the main cashmere-producing province of Kerman, cashmere is shorn by nomads in different months of spring using double-blade scissors called docard. In a study with 30 nomad settlements chosen at random within $\pm 20 \mathrm{~km}$ of Baft city in Kerman Province, it was revealed that $52 \%$ and $30 \%$ of nomads sheared their goats in May and April while $11 \%$ and $7 \%$ do so in March and June, respectively (Ansari-Renani et al. 2013b). In early spring (March), cashmere goats start a sequential, bilaterally symmetric pattern of shedding their fine undercoat fibres commencing on the neck, chest and shoulders then spreading to the back and rump. Shed fibres remain some time entangled in the rest of the fleece but eventually fall off the animal.

\section{Cashmere shedding and time of fibre harvesting in nomadic areas}

In terms of harvesting maximum weight of fibre, the optimal time for a single shearing would be at the end of winter season in January or early February at the peak of follicle inactivity or before onset of shedding. At the time of cashmere harvesting, animals are in their poorest body conditions after the cold weather and very limited feed availability (Ansari-Renani et al. 2013c). It is important from the point of animal welfare that some hair is left on the skin after fleece harvesting as this hair provides an essential protective layer (McGregor 1988) against adverse weather conditions. Use of shearing methods, either machine or hand shearing which removes the entire fleece, poses a serious threat and is inconceivable under such conditions. In addition, the presence of unshed long coarse guard hair in the fleece helps fibre loss once shedding has begun by acting as a physical barrier restraining the cashmere in its position (McGregor 1988). This allows owners to time their fibre harvesting, either using combs or by collecting the clumps of hair retained in the fleece. Use of combs reduces further fibre loss during the shedding season.

\section{Cashmere marketing}

Cashmere-producing areas included in the marketing study

The nomadic pastoralists who produce cashmere in Iran are differentiated by herd size, income levels and grazing management. About half of nomad households in Baft area own 200 to 300 Raeini goats, with $23 \%$ and $30 \%$ of families owning less than 200 and more than 300 goats, respectively (Ansari-Renani et al. 2013b). Livestock is their largest asset and predominates as a source of livelihood and supply of food. The nomadic pastoralist system here, as elsewhere, is characterized by low population density, 
self-reliance for basic needs, movement of livestock between grazing sites in different seasons and weak linkages to markets and public services. Rangeland is considered as the main source of grazing and depends on seasonal rainfall. In the previous decades, these households have been able to operate at the margin only due to the implicit cost-free public land and water and restocking of goats after livestock numbers are devastated in harsh winters and drought seasons.

\section{Nomad cashmere producers}

A competitive advantage for the cashmere industry in east Iran is its access to raw cashmere, which is produced by low-income nomad herders in eastern Iran and bordering Afghanistan. There is a large seasonal variation of supply, demand and price of cashmere. Cashmere harvesting and buying takes place over a short spring period in nomadic areas. In-depth open interviews and group discussions conducted in the eastern regions with key industry actors revealed that $80 \%$ of cashmere is sold to dealers from April to June and $20 \%$ of the remaining is sold in July. While $62 \%$ of nomads sell their cashmere in one period of time, $22 \%, 11 \%$ and $5 \%$ sell their harvested cashmere at two, three and four different intervals, respectively (Ansari-Renani et al. 2013b). All nomad goat owners sold the whole fibre, undehaired, containing both rough outer hair and the inner fine cashmere, to local or travelling merchants or traders. The raw cashmere is unsorted and sold at lower prices than could be expected if the cashmere was sorted at source into quality classes.

Results from previous studies indicate that $30 \%$ of cashmere is lost during shedding season and if not harvested would be wasted (Ansari-Renani et al. 2011a).

Nomad cashmere producers do not receive seasonal price information on cashmere through reliable and upto-date sources. Results indicated that $41 \%$ and $32 \%$ of nomads receive scattered marketing information from traders or neighbouring farms, respectively, while $14 \%$ and $13 \%$ received their information from associations and markets, respectively (Ansari-Renani et al. 2013b).

Nomads market their cashmere on an 'eye-ball' basis: transactions are made between individuals and agreements reached after one-to-one bargaining between buyers and sellers. The reason for 'eye-ball' marketing is the lack of infrastructure for measurements of the cashmere.

There have been some changes in marketing from time to time and in various parts of the country, but in general no drastic changes for some years. Perhaps the biggest change has been the general trade practice whereby most of the warehousing of cashmere is done near the source of supply. Another change has been the increase in direct buying from nomads by processors or manufacturers. According to the interviewed nomad cashmere producers, the local buyers collect cashmere and either sell to a merchant in town or store in their own facility. Most dealing of this kind is with nomads having a limited number of goats.

The nomads stress the fact that life in the nomadic region has become very hard due to the economic situation. Nomads noted that their livestock do not produce enough milk, cashmere and meat to sustain them throughout the year. Thus, the nomads have to consume a larger part of the yield of their capital (livestock) than they would otherwise have done. This can have dire consequences, since they constantly are under threat of losing part of their flocks due to environmental factors, and smaller flock sizes make it more difficult to survive a disaster by recovering flock sizes during good years.

The nomadic pastoralists are declining in number and resort to all means of occupation, exacerbated due to efforts of the governments to sedentarize them as well as due to severe environmental conditions such as droughts which force the nomads to search for wages in urban areas.

\section{Middlemen}

The important agencies involved in getting the cashmere from the nomad cashmere producers to the manufacturers are the country buyer, the country assembler or cashmerewarehouse handler or dealer or combination, local cashmere pools, the commission merchant and the broker.

Many local dealers or purchasers of small lots of cashmere may be junk dealers or feed-store operators. Many of these dealers specialize in not only cashmere but other products of the region such as furs, hides, pelts, iron, poultry or possibly livestock. Usually, the cashmere is sold ungraded, at a flat price. However, if some preparation has been done on the fleeces, they may be roughly sorted. Cashmere is loosely packed by middlemen in tall narrow polyester bags $(90 \times 36 \times 30 \mathrm{~cm})$, weighing about 100 to $150 \mathrm{~kg}$ per bag. Figure 1 is an illustration of packing cashmere in sacks.

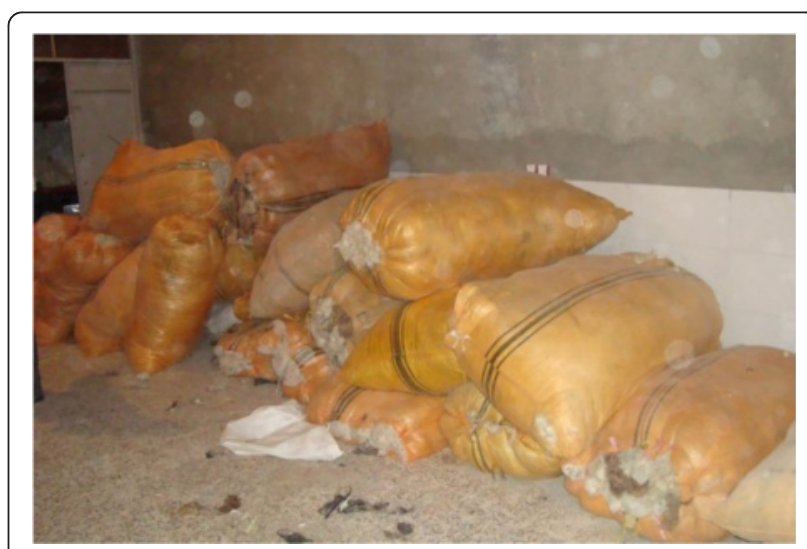

Figure 1 Cashmere is kept in polyester sacks and stored in local warehouses near the production site. August 2011 
Cashmere pools have operated for years and are becoming more numerous in the eastern cashmere-producing provinces. These pools are usually made up of many growers' clips in a nearby locality, which pool their cashmere in one central location and have buyers come and make a price offer. Sometimes the cashmere may be sorted, but more often they are not.

In nomadic areas, a cashmere buyer may be a local man living in the rangeland all year round, or he may be a man sent directly from the country buyer. Some large purchasers of cashmere during the last several years have set up permanent buying organizations at central locations in the nomadic areas of eastern cashmeregrowing regions. Thus, the buyers are closer to the source of cashmere and effect a saving here and also through direct shipments to mills. Local buyers generally work on commission; this is based on the amount of cashmere purchased, whereas some buyers are normally paid a salary and their travelling expenses. Some eastern cashmere middlemen have purchasing orders from different merchants or mills for specified quantities of various types of cashmere. This is done especially by lager mills, who desire to purchase in advance cashmere suitable for their sales requirements, and in such cases, the buyer works on a commission.

Cashmere producers in the nomadic areas sell unsorted raw cashmere at lower prices than could be expected if the cashmere was sorted at source into quality classes. Nomad cashmere producers do not receive seasonal price information on cashmere through reliable and up-to-date sources.

After harvesting, cashmere is bought directly from the nomads by middlemen and the fibre is stored in warehouses, according to colour and fineness. A basic problem that operators of cashmere warehouses have in some years, especially during drought, is to obtain adequate volumes of cashmere for efficient handling. This involves consideration of the sources and availability of potential supplies, the competition from others, as well as transportation facilities and costs. With sufficient volumes of cashmere available, other problems include securing at reasonable costs adequate protection from losses by fire and other hazards, obtaining and maintaining suitable facilities and equipment for efficient rendering of essential warehouse and related services, securing adequate information concerning the quality and commercial value of the cashmere handled, and maintaining suitable contacts with market outlets to dispose of the cashmere qualities handled.

Raw sorted cashmere from gathering centres is sent in bales by trucks or by train to Bandar Abbas and Khoramshahr, the main Iranian exporting ports in the Persian Gulf. From these ports, baled raw cashmere is shipped to Italy, Belgium and England. However, about one third of raw Iranian cashmere stock goes to Semnan and Mashad for

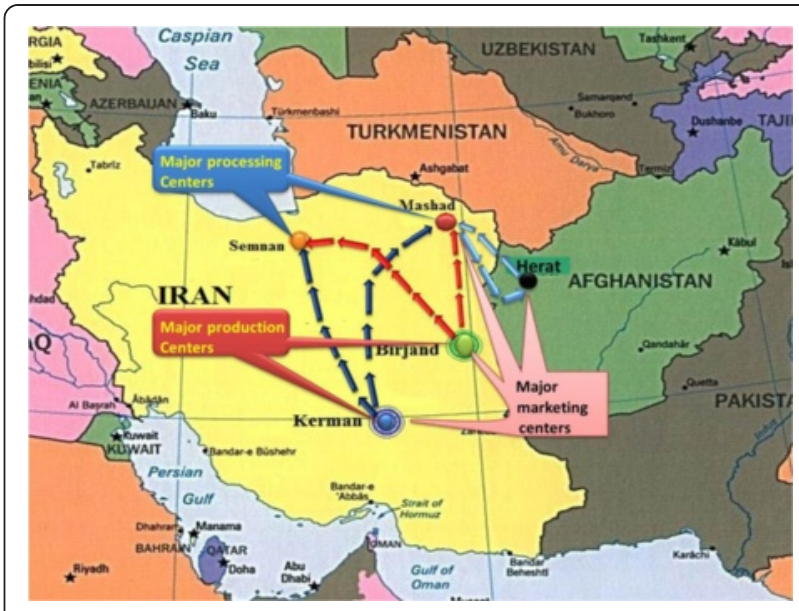

Figure 2 Major production, marketing and processing centres of Iranian cashmere

processing. Processed cashmere also finds its way to the same Persian Gulf ports to be exported to other countries.

The manufacturer or national buyer sends his representatives through the small towns to dealers or to local warehouses to buy the cashmere. The cashmere purchased may then be shipped to the main trading centre such as Baft, Sirjan, Mashad and Birjand (Figure 2).

These centres have long been considered the leading markets in Iran. Since the 1950s, the market established itself near the production areas, with more shipments going directly to mills. Mashad continues as the main centre of the cashmere industry, as most of the large manufacturers, exporters, dealers, selling agents and warehouse dealers are located there. Khorasan Razavi Province, with Mashad city as its centre, having a border with Afghanistan, also plays an important role in the processing and marketing of Afghan cashmere industry. Figure 3 illustrates the main market channels of Iranian cashmere.

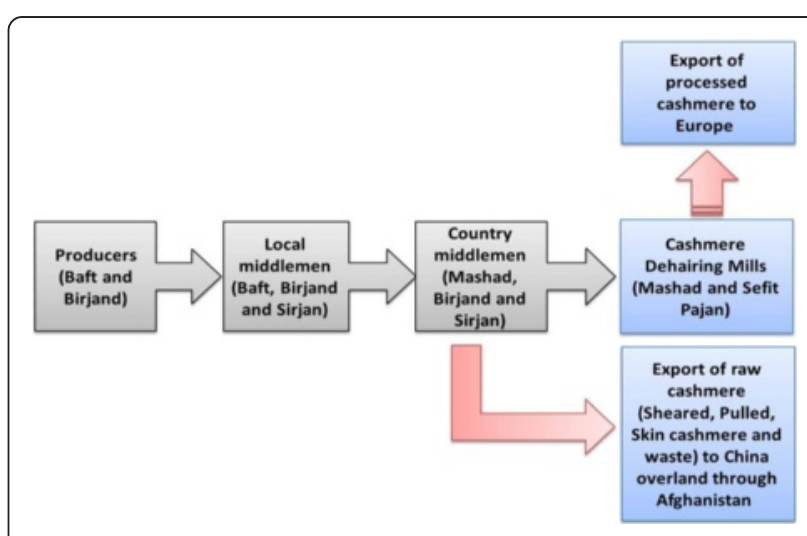

Figure $\mathbf{3}$ The principal channels for the gathering and rough sorting of raw cashmere in Iran 


\section{Cashmere mills and export market}

The local mills in Mashad and Semnan scour and dehair about $30 \%$ of the locally produced cashmere and $29 \%$ of Afghanistan's clip. Almost all Iranian processed dehaired cashmere is exported to European countries for making garments. Distribution pathways of raw cashmere shipments from Iran to the major importing countries are presented in Figure 4 as cashmere is exported to China and European countries. World trade of Iranian cashmere is accounted for principal cashmere-importing countries of China, Britain, Belgium and Italy.

China is not only a heavy producer but also a major importer of Iranian raw cashmere. China also imports significant volumes of cashmere in other various forms. Access to imported cashmere provides another important source of competitive advantage to Chinese processors, especially as the trade partners are located in the relatively nearby regions of Pakistan, Afghanistan and especially Mongolia.

Afghan cashmere has traditionally been exported to Iran for early-stage processing and then exported for final processing (De Weijer 2008). However, with decreased purchases from Europe after the 2008 global financial crisis, Chinese buyers have become very active in Afghanistan (Cashmere Fibres International 2009).

Iranian traders play an important role in the export of Afghanistan raw and dehaired cashmere to Belgium for disinfection, which may explain why most of the cashmere production and trading is still taking place in the northwest west of Afghanistan (Altai Consulting Ltd 2005) close to Mashad city in Iran (Figure 2). Verviers, in Belgium, used to be the main market centre for cashmere, as it was suited in the core of the textile centre in Europe. Nowadays, the role of Verviers as the main market place has diminished, but for Afghan cashmere, Verviers is still a major destination through Iran.

Cashmere from Afghanistan is being processed in Mashad scouring and dehairing facilities and then transported to Europe through Iran. Iranian processors have

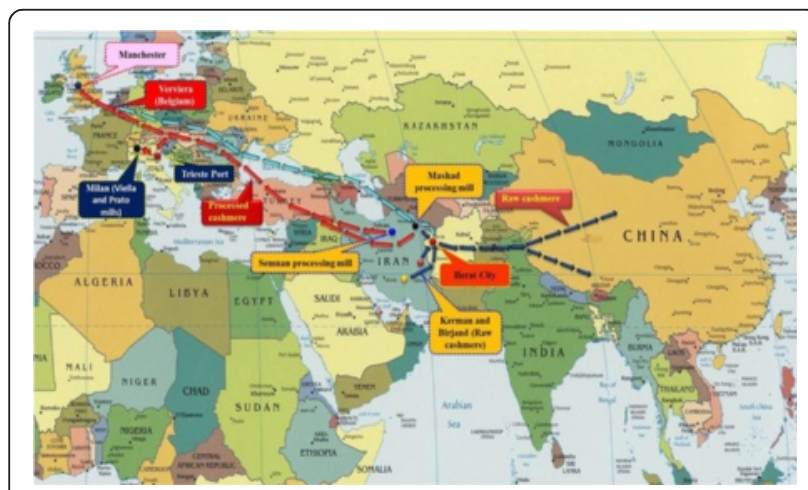

Figure 4 Distribution pathways of raw cashmere shipments from Iran to the major importing countries established relations with certain Herati exporters in Afghanistan, who supply these dehairing facilities.

\section{Cashmere prices and valued addition along the marketing chain}

Increased demand from Chinese processors, along with supply-side constraints, has increased international cashmere prices. Figure 5 shows that between 2002 and 2010, annual average prices increased by $10 \%$ in China and $11 \%$ in Mongolia and Iran. These price signals triggered an expansion of the Mongolian goat herd at an annual average rate of $10 \%$ over the period, to reach 20 million head (the vast majority of which are cashmere goats). while China has by far the world's largest goat population (Food and Agriculture Organization of the United Nations 2014); statistics for China as a whole do not distinguish between cashmere and meat goats. While cashmere goat numbers have plateaued in pastoral and semi-pastoral counties, cashmere production in China increased (Schneider 2012).

Pastoral and semi-pastoral regions of Iran produce almost $75 \%$ of all Iran's cashmere. Only one fifth of the Iranian goat population is cashmere-producing; however, based on household surveys and interviews conducted, there is indication that households have clear incentives to switch from the production of hairy goats to cashmere goats and, of the cashmere goat breeds available, maximize profits by selecting Raeini and Birjandi breeds of goats over the hairy-type (scrub) goats which have a population of more than 18 million head. This population of hairy goats also have an undercoat of downy fibre that is classed as 'cashmere'. While only a very small quantity of this down is produced per animal, it can be worthwhile for farmers or traders to comb or fellmonger (removing fibre from the surface of the skin using hairremoving chemical agents) the down from skins, especially when prices are high. This cashmere can be used

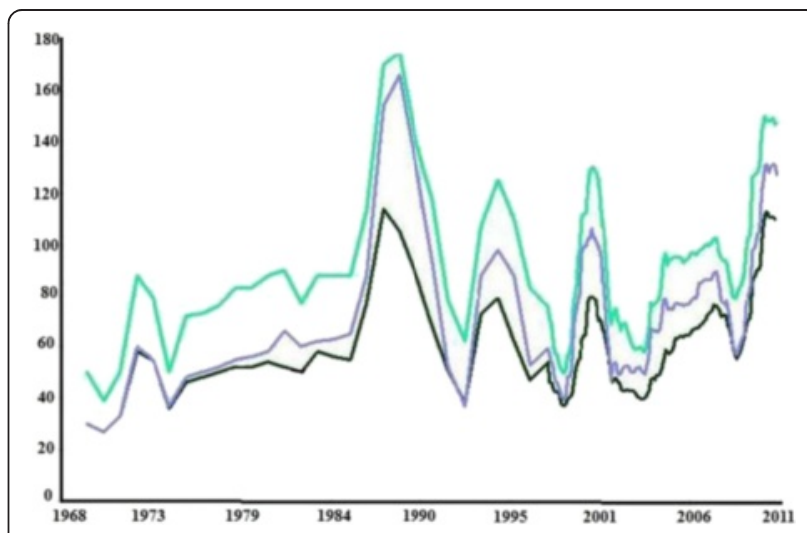

Figure 5 Cashmere prices of China, Mongolia and Iran over time. Chinese cashmere is sold at higher prices than Mongolian (second highest) and Iranian cashmere (adapted from Schneider 2012) 
to produce low-value products and for blending to produce 'cashmere' products. If no price-grade differentials are paid for the cashmere - as is the case under the current Iranian marketing system - then due to their higher cashmere yields, Raeini cashmere breeds of goats are more profitable than hairy goats.

Production of cashmere in Iran takes place in selected provinces. At the lowest section of the value addition chain, considerable potential exists to expand production of cashmere to goat farms in other provinces.

Such expansions should be done with care over time, as 'quick fixes' such as crossing with very high producing exotic goats tend to decrease fibre diameter rapidly by reducing staple length and fibre curvature creating short, flat fibre. The way to restore the quality of Iranian cashmere is through herd selection and superior genetics. There is scope within the national herd to increase fibre yield without reducing fibre quality and thus increasing financial returns to farmers.

Higher export earnings from cashmere could help to boost the income of a large number of Iranian cashmere nomad herders, if more of the value was passed on to the producers, many of whom represent the poorest segments of Iranian society. There are ambiguities, however, about the sustainability of cashmere production as a livelihood for nomad and smallholder households.

Value addition takes place at different stages of the production and market chain. Most of the power within the value chain resides with the privately owned processing firms and retailers; herders are the largest group of actors but perhaps the least powerful. Lack of market information makes herders vulnerable to exploitation by processors and traders, and without price differentials between high-quality cashmere and other grades, herders lack incentives to invest in improving their herds. In another study with cashmere goats in the Pamir mountain districts of Tajikistan, McGregor et al. (2011) indicated that villagers need objective and up-to-date information on commercial prices for better quality cashmere, to motivate them to select finer bucks.

Currently, Iran is engaged with cashmere production, harvesting, followed by industrial scouring, dehairing, carding and combing. These are the low to medium added cashmere value activities at the downward section of the chain. Figure 6 shows schematically the cashmere value chain of harvested cashmere from the herder, assembly and exporter levels. Despite $50 \%$ unavoidable loss from washing and removing the outer guard hair from cashmere, the value is doubled by weight after this stage. Thus, after scouring and dehairing, $1 \mathrm{~kg}$ of raw greasy cashmere is transformed to four times its initial value. Major burr and grease seed contaminants of cashmere result in serious price penalties and so do the guard hair levels. Any undesirable contaminant, which will either

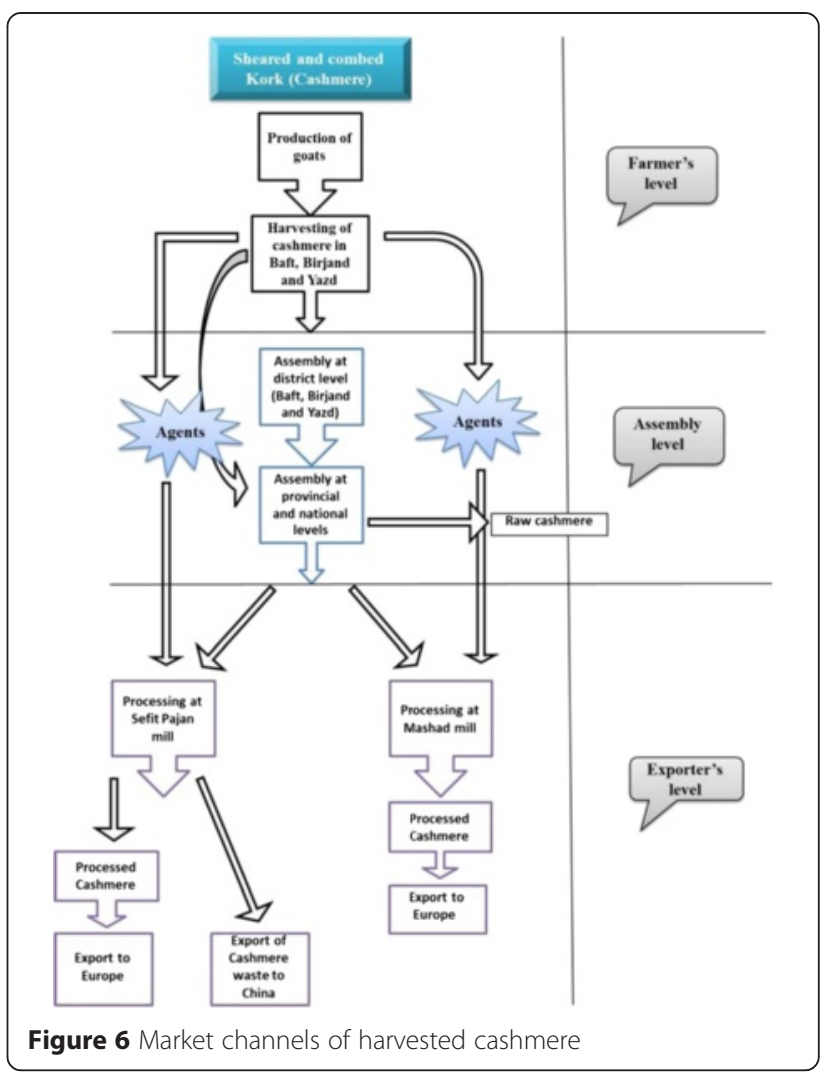

affect the quality of the final product or will have to be removed, reduces the economic value of the cashmere. Burrs or excessive vegetable matter in the fleece also have to be removed. Urine and certain types of soil and vegetable matter contain substances which stain cashmere permanently. These affect the dyeing and value of the cashmere and the quality of the final product. Precautions must

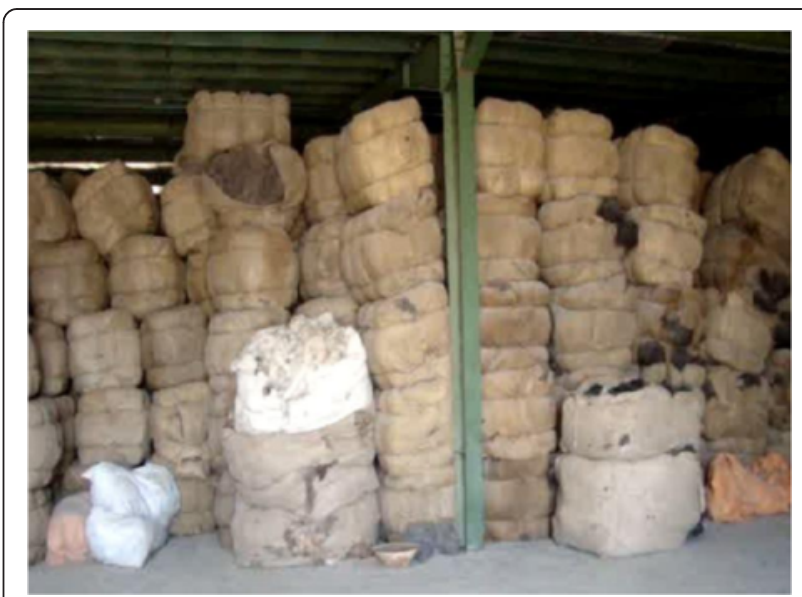

Figure 7 Bales of different colours of cashmere transported from production sites and kept in warehouses at Mashad processing factory. October 2011 

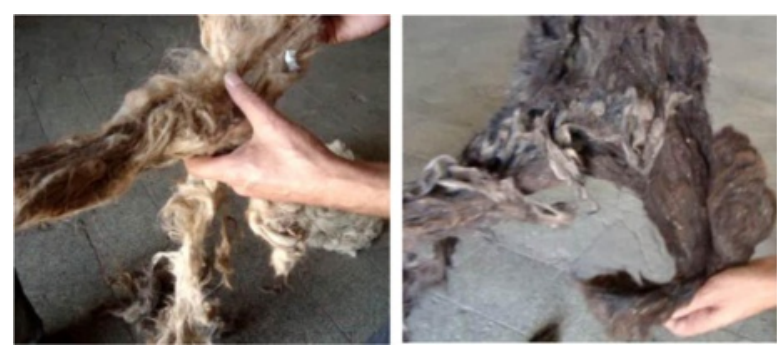

Figure 8 Skirting black and white Birjandi and Raeini goat fleeces from South Khorasan and Kerman Provinces, respectively. Sefit Pajan processing mill in Semnan Province. October 2011

be taken to limit such stained fibre entering the market chain, particularly from urine stains.

A producer training programme on combing cashmere would add the commercial value of their cashmere. Commercially recoverable cashmere yields are higher from combed rather than shorn fleeces, making combed cashmere more attractive to textile processors. Very low yielding fleeces need extra dehairing and have a higher residue of coarse fibres left after dehairing. The extra processing also tends to cause more fibre breakage, leading to lower final product prices and less added commercial value as a result of shorter fibre length and hence lower yarn quality.

\section{Cashmere processing}

Major processing centres of Iranian cashmere are Semnan and Mashad cities (Figure 7). Dehaired cashmere is packed in bales and exported to other countries.

\section{Cashmere sorting}

Raw fibre reaches the mills in the form of fleeces which are packed and transported in jute or polyester sacks. On being brought out from the sacks on the sorting floor, the Lapich (twisted) fleece is skirted and opened widely (Figure 8).

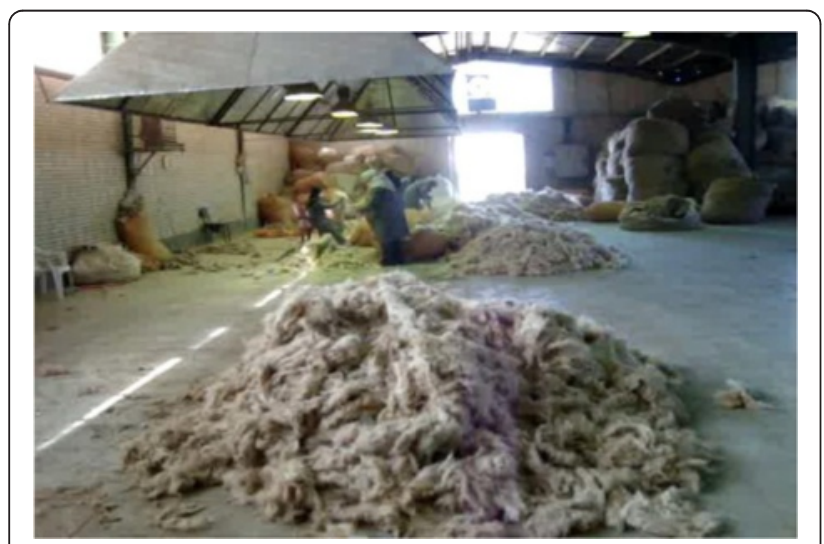

Figure 9 Cashmere sorting area. A proper light is necessary for sorting. Note the piles of unsorted fleeces in the background. Sefit Pajan processing mill in Semnan Province. October 2011

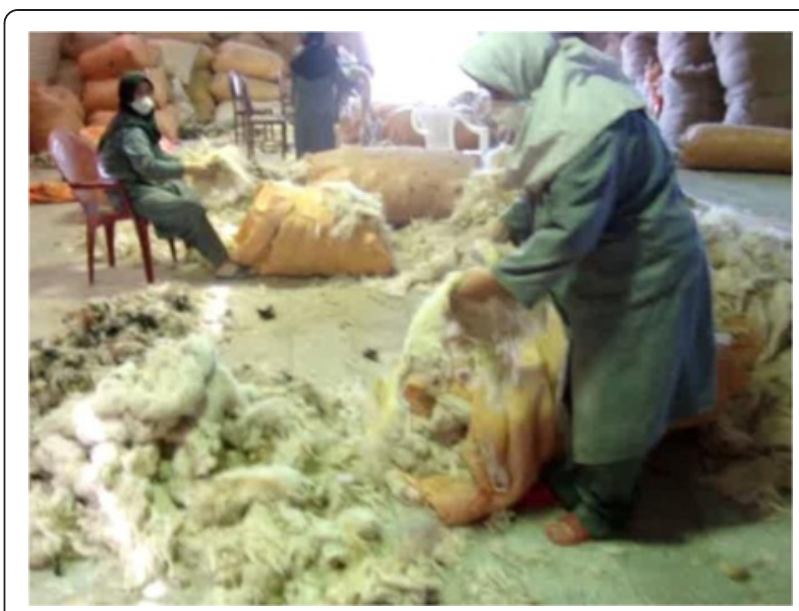

Figure 10 Two cashmere women sorters at work in Pajan processing mill in Semnan Province. October 2011

In the factory, cashmere is sorted according to fineness, length, soundness, colour and the amount of vegetable matter, enabling the manufacturer to produce out of each sort the yarn or fabric for which it is best suited. In Iran, special care is taken with sorting. A proper light is necessary (Figure 9); experience has shown that poor illumination causes the sorter to grade the cashmere too fine and in direct sunlight the fibre appears coarser to the sorter. Skirting is the next step, involving removal of any objectionable sorts from the fleece (Figure 10).

The inferior portions or objectionable sorts are heavy shrinking parts such as tags, leg pieces, neck pieces, bellies, locks and stained portions. These portions are sorted into individual bins or containers (Figure 11).

\section{Dusting or opening}

Cashmere is generally run through a double-cylinder opener for dusting or opening especially dirty, long and coarse cashmere (Figure 12).

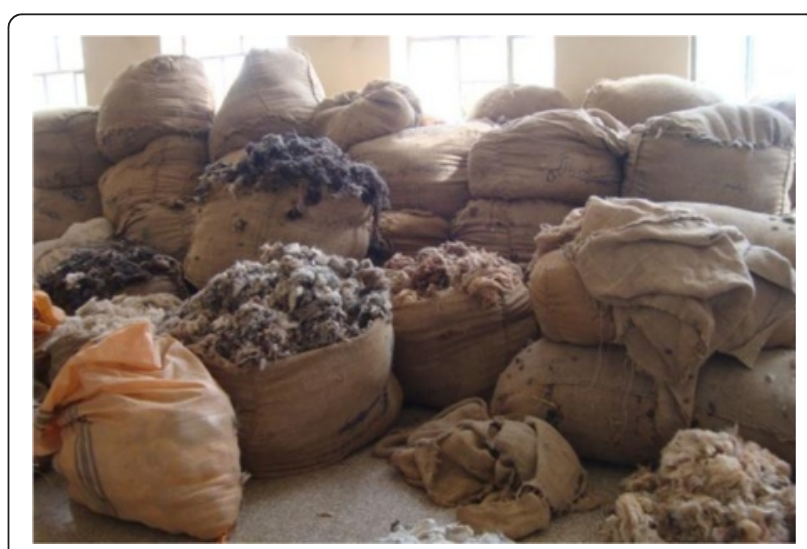

Figure 11 Piles of sorted black, white and fawn Birjandi and Raeini goat fleeces from South Khorasan and Kerman Provinces. Mashad processing mill in Khorasan Razavi Province. October 2011 


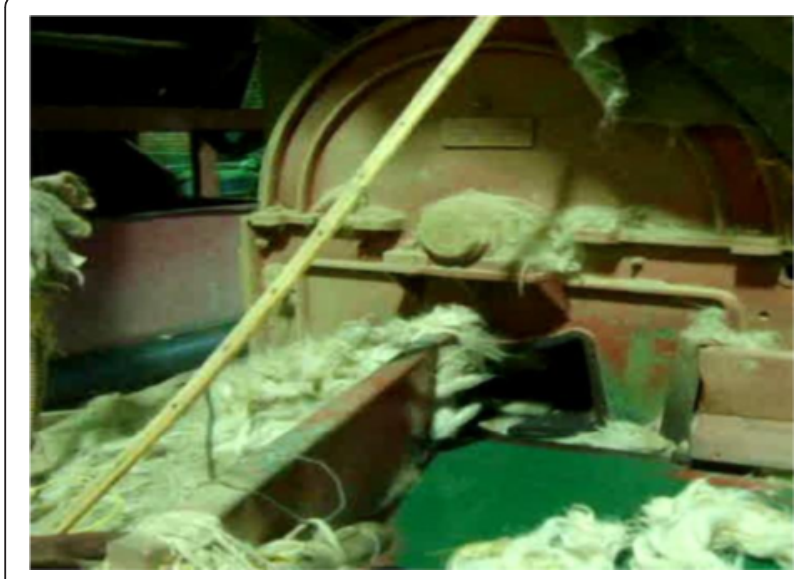

Figure 12 Double-cylinder cashmere opener. Sefit Pajan processing mill in Semnan Province. October 2011

\section{Cashmere scouring}

The removal of impurities in raw cashmere is one of the more intricate and important operations in the manufacture of cashmere material. In Iran, the scouring of greasy cashmere is accomplished by the 'aqueous scouring process' method in a series of individual vats or bowls through which the cashmere is propelled by

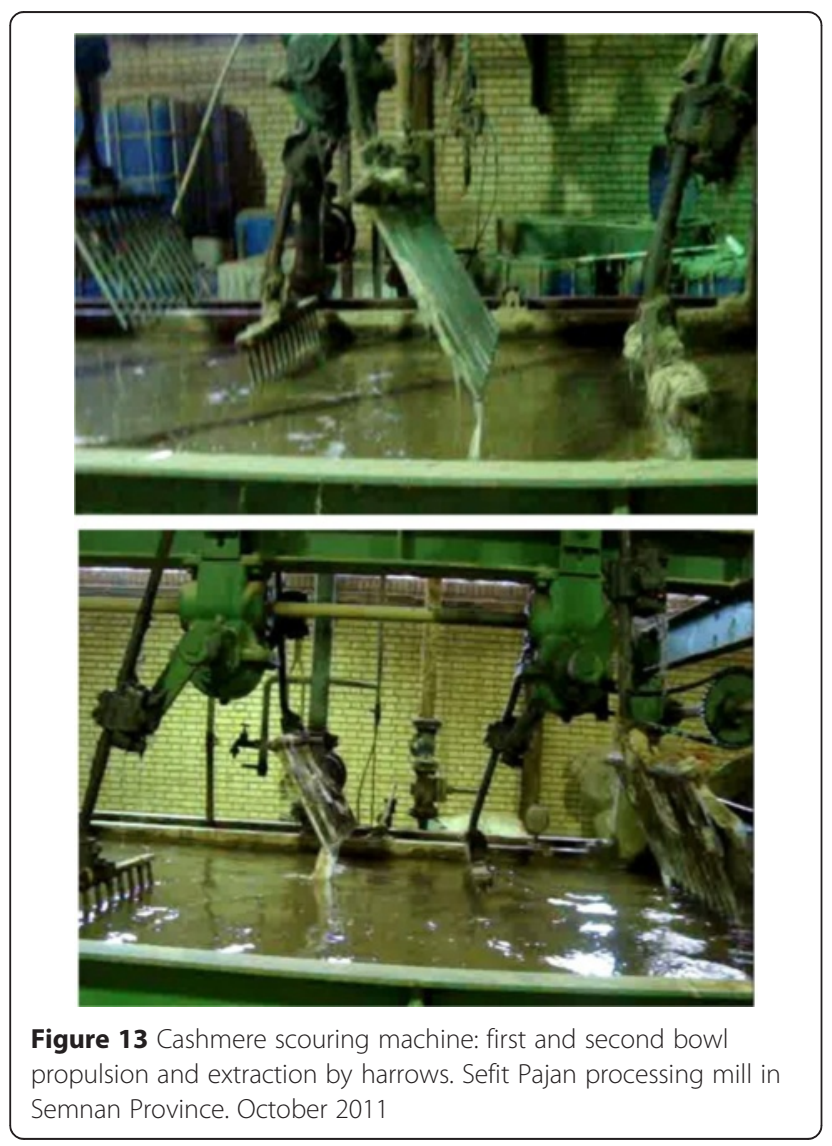

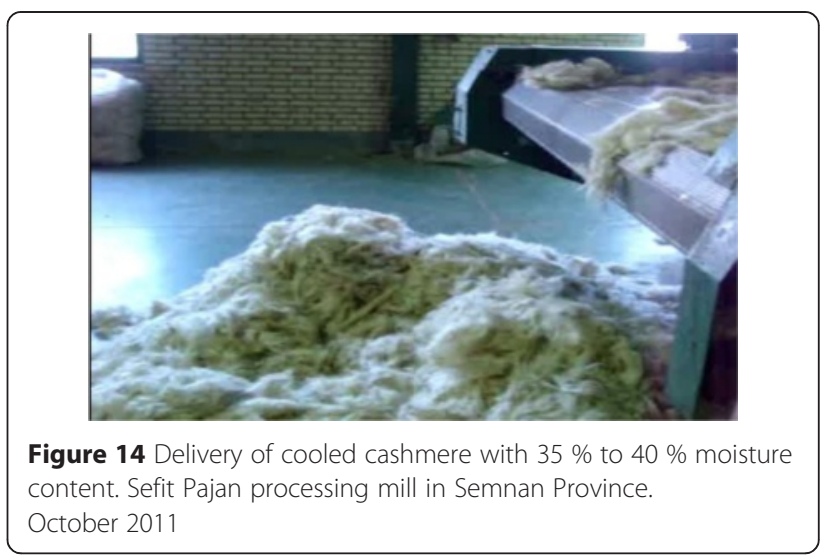

mechanical racks with intervening squeeze rolls (Figure 13).

\section{Cashmere drying}

The washed cashmere should enter the dryer with moisture content around $40 \%$ or even lower. The cashmere is carried on a single conveyer (Figure 14) where it is subjected to heated air. Dried cashmere is then kept in a humid conditioning room which maintains the moisture content of cashmere at a level needed for the carding process.

\section{Burr picking}

The primary object of burr picking (Figure 15) is to remove by mechanical means all the vegetable matter possible before the stock is passed onto the cards.

\section{Garneting}

Garneting is the thorough breaking up of the waste and its return to a fibrous condition so that it can be used in mixes with virgin cashmere. The material is fed to the machine (Figures 16 and 17) by hand.

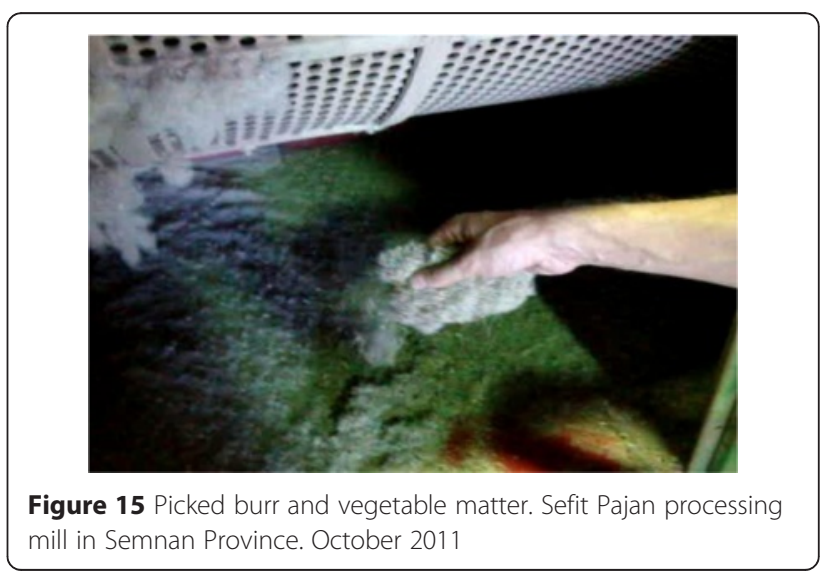




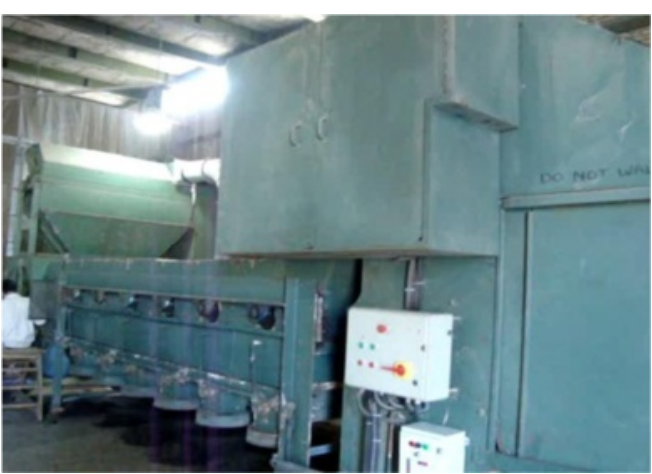

Figure 16 Garneting machine. Short cashmere fibre is manually fed into the machine. Mashad processing mill in Khorasan Province. October 2011

\section{Blending or mixing}

The object of blending or mixing is the amalgamation of the various materials, as thoroughly as possible (Figure 18).

\section{Oiling the stock}

Cashmere stock must be lubricated to minimize breakage of the cashmere fibres in opening processes such carding, as well as to reduce fly, waste and static electricity in carding.

\section{Carding}

Carding constitutes the last operation in which the cashmere fibres can be properly opened.

\section{Dehairing}

In a modified Iranian cashmere processing, the guard hair is removed by carding and dehairing (Figure 19) ending up with cashmere fibre (noil) free of coarse hair.

\section{Cashmere baling}

Essentially, vacuum pressing is the common method in the Mashad processing mill; the method is to fill a

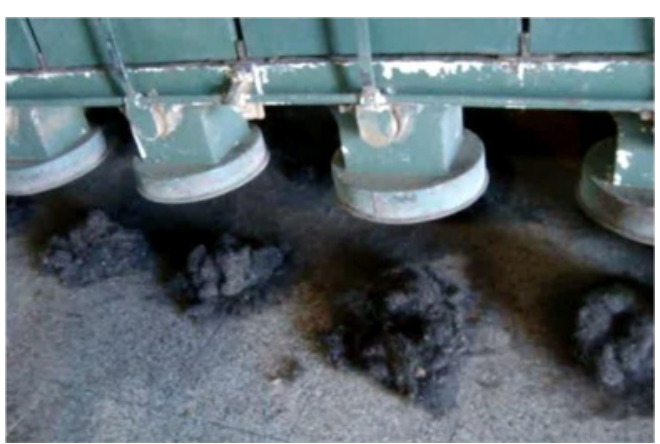

Figure 17 Guard hair and cashmere waste is discarded from the exit valves beneath the garneting machine. Mashad processing mill in Khorasan Razavi Province. October 2011

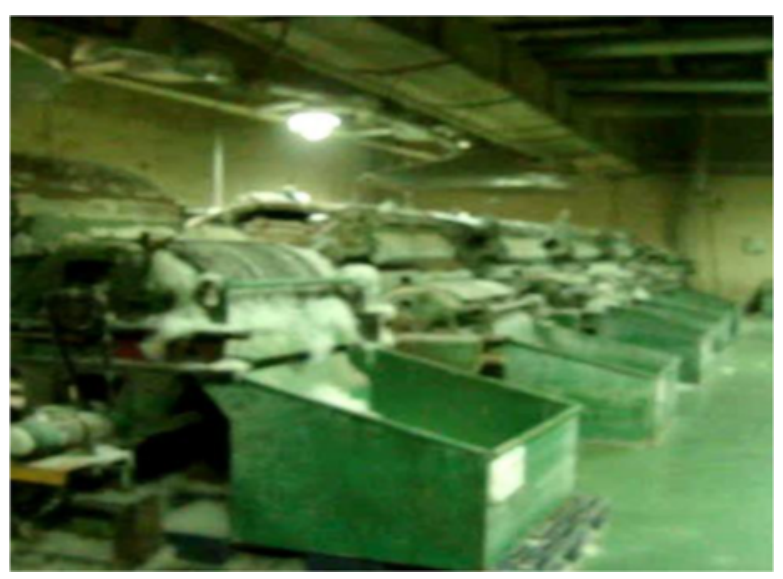

Figure 18 Blending installations. Sefit Pajan processing mill in Semnan Province. October 2011

flexible airtight container such as a polythene bag with the desired weight of cashmere and then to evacuate the air.

\section{Conclusion}

Iranian nomads are not aware of world market prices for different cashmere quality classes. As a result of the marketing system, nomads do not achieve good prices and have little incentive to produce better quality cashmere. Poor marketing infrastructure, lack of access to formal credit sources and lack of standardization and grading are
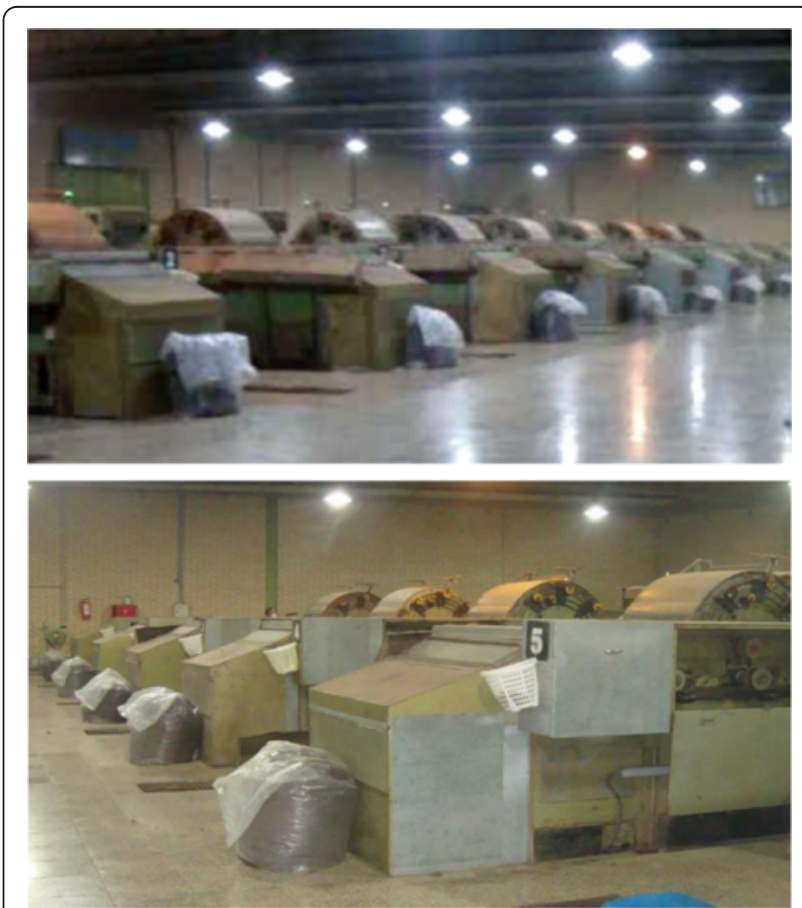

Figure 19 Cashmere dehairing machines. Mashad processing mill in Khorasan Razavi Province. October 2011 
major constraints affecting the value addition of cashmere marketing. All Iranian cashmere is exported without major value addition. To achieve more value addition, attention must be given to improvement in market information and intelligence and formulation of new marketing and processing policies.

\section{Competing Interests}

The author declares that he has no competing interest.

\section{Authors' contributions}

HRAR acquired, analysed and interpreted the data and wrote the manuscript.

\section{Authors' information}

HRAR is an associate professor in the Animal Science Research Institute (ASRI) of Iran who has conducted research on nomadic livestock management and smal ruminants with particular emphasis on fibre production.

Received: 10 May 2015 Accepted: 21 September 2015

Published online: 09 November 2015

\section{References}

Allain, D. 1992. Biology and characteristics of goat fibers. In New developments in goat husbandry for quality production. Seminar proceeding held at Lisbon, Portugal. 27-29 October, 1992, ed. H Galbraith, 22-35.

Altai Consulting Ltd. 2005. Market Sector Association - SME development, Chapter V, "Cashmere". Altai Consulting, for UNDP Kabul

Ansari-Renani, HR. 2001. Seasonal follicle cycle and fibre shedding of Raein cashmere goats. J. Pajou. and Sazan. Ministry Agriculture, Iran 14(4): 23-25.

Ansari-Renani, HR. 2004. The hair follicle group and skin follicle population of Iranian cashmere and mohair producing goats. Proceeding of first animal and fisheries congress, 867-871. Iran: Tehran University.

Ansari-Renani, HR. 2012. Fiber quality of Iranian carpet-wool sheep breeds. Journal of Animal Science and Technology 35(3): 179-184.

Ansari-Renani, HR. 2013. Cashmere quality of Iranian goat breeds. Journal of Animal Science and Technology 35(3): 179-184.

Ansari-Renani, HR, M Salehi, Z Ebadi, S Moradi, HR Baghershah, Ansari -Renani MY, and SH Ameli. 2011a. Determination of hair follicle characteristics, density and activity of Iranian cashmere goat breeds. Small Ruminant Research 95: 128-132.

Ansari-Renani, HR, S Moradi, HR Baghershah, Z Ebadi, M Salehi, SM Seyed Momen, and MY Ansari-Renani. 2011b. Determination of wool follicle characteristics of Iranian sheep breeds. Asian Australasian Journal of Animal Science 24(8): 1173-1177.

Ansari-Renani, HR, JP Mueller, B Rischkowsky, SM Seyed Momen, O Alipour, M Ehsani, and S Moradi. 2012. Cashmere quality of Raeini goats kept by nomads in Iran. Small Ruminant Research 104: 10-16.

Ansari-Renani, HR, JP Mueller, B Rischkowsky, and S Moradi. 2013a. Cashmere in Iran. ICARDA-ASRI, 4-5

Ansari-Renani, HR, JP Mueller, B Rischkowsky, SM Seyed Momen, and S Moradi. 2013b. Nomadic pastoralism in southern Iran. Pastoralism: Research, Policy and Practice 3: 11.

Ansari-Renani, HR, JP Mueller, B Rischkowsky, SM Seyed Momen, M Ehsani, S Moradi, et al. 2013c. Observations on the efficiency of using different cashmere combs. Small Ruminant Research 114: 220-224.

Carter, HB, and WH Clark. 1943. The hair follicle group and skin follicle population of Australian Merino sheep. Australian Journal of Agricultural Research 8: 91-108.

Cashmere Fibres International. 2009. Market report - November, 2009.

De Weijer, F. 2008. Cashmere value chain analyses Afghanistan, 38. USAID: Accelerating Sustainable Agriculture Program.

Food and Agriculture Organization of the United Nations. 2014. WWW.FAOSTAT.com.

McGregor, BA. 1988. Production of cashmere in northern China. Study tour report No. 134. Victoria: Department of Agriculture and Rural Affairs.

McGregor, BA. 2007. Cashmere fibre crimp, crimp form and fibre curvature. International Journal of Sheep and Wool Science 55(1): 8.

McGregor, BA, and R Postle. 2004. Softness and other fibre attributes of commercial cashmere textiles from China and other origins of production. In Proceedings of the Textile Institute 83rd World Conference, May 23-27, Shanghai, 372-375.
McGregor, BA, C Kerven, and S Toigonbaev. 2011. Sources of variation affecting cashmere grown in the Pamir mountain districts of Tajikistan and implications for industry development. Small Ruminant Research 99: 7-15.

Mitchell, RJ, K Betteridge, MP Gurnsey, RAS Welch, and AJ Nixon. 1991. Fibre growth cycles of cashmere-bearing reproducing does in Southern Hawkes Bay, New Zealand, over 30 month period. New Zealand Journal of Agricultural Research 34: 287-294.

Nixon, AJ, MP Gurnsey, K Betteridge, RJ Mitchell, and RAS Welch. 1991. Seasonal hair follicle activity and fiber growth in some New Zealand cashmerebearing goats (Capra hircus). Journal of Zoology (London) 224: 589-598.

Petrie, OJ. 1995. Harvesting of textile animal fibers. Rome: Food and Agricultural Organization of the United Nations. No. 22.

Ryder, ML. 1966. Coat structure and seasonal shedding in goats. Animal Production 8: 289-302.

Schneider, GS. 2012. Market indicators. http://www.gschneider.com/indicators/ index.php.

\section{Submit your manuscript to a SpringerOpen ${ }^{\circ}$ journal and benefit from:}

- Convenient online submission

Rigorous peer review

- Immediate publication on acceptance

- Open access: articles freely available online

- High visibility within the field

- Retaining the copyright to your article

Submit your next manuscript at $>$ springeropen.com 